\title{
Adherence in Adolescent and Young Adult Kidney Transplant Recipients
}

\author{
Bethany J. Foster ${ }^{*}, 1$ and Ahna L.H. Pai ${ }^{2}$ \\ ${ }^{I}$ Montreal Children's Hospital \& McGill University, Canada \\ ${ }^{2}$ Cincinnati Children's Hospital Medical Center \& University of Cincinnati College of Medicine, Canada
}

\begin{abstract}
Poor adherence to immunosuppressive medications may be the most important barrier to long term graft survival. An understanding of medication adherence and its determinants is critical to addressing this important problem. In this paper, we will review the different ways in which adherence may be compromised, summarize the evidence that young people constitute a particularly high risk group, and consider the consequences and impact of poor adherence. We will also review the determinants of adherence, including characteristics of the patient and family, the treatment regimen, the healthcare team and its organization, and the healthcare system. We will highlight the most common barriers to adherence identified by young people, and consider different methods of measuring adherence, along with the advantages and limitations of each. Finally, we will consider possible intervention strategies to improve adherence in young people.
\end{abstract}

Keywords: Adherence, adolescence, compliance, emerging adulthood, pediatric, renal transplant.

\section{INTRODUCTION}

Poor treatment adherence is one of the most important obstacles to graft longevity in kidney transplantation [1-3]. Effective immunosuppressive medications cannot prevent rejection if they are not consumed every day on a strict schedule. Similarly, graft dysfunction signalling possible rejection cannot be detected and treated early without regular monitoring of serum creatinine. Although transplant recipients of all ages may have difficulty adhering to a strict treatment plan, adolescents and young adults are at particularly high risk for poor adherence [4-6]. Not coincidentally, young people also have the highest risk for graft failure of any age group [7].

Adherence is defined as the extent to which an individual's behavior coincides with medical or health advice [8]. This definition underscores the socially constructed nature of adherence by focusing on the correspondence between patient behaviors and the prescriptions, recommendations, and/or advice from health care providers. As such, the interchange between patients and providers is critical to optimizing adherence. The World Health Organization (WHO) definition, emphasizes the need for the patient and provider to have an agreed upon treatment plan [9]. Although, we concur that the patient's agreement is ideal, this is difficult to objectively verify. Therefore, the alternate definition is used for the purpose of this review. Of course the goal of any treatment plan is to optimize outcomes. With this in mind, satisfactory adherence has been defined as sufficient concordance between the prescribed treatment plan and the patient's behaviour such that outcome is unaffected by any deviations

*Address correspondence to this author at the Montreal Children's Hospital, Division of Nephrology, E-222, 2300 Tupper Street, Montreal, QC H3H 1P3, Canada; Tel.: 514-412-4461; Fax: 514-412-4359;

E-mail: Bethany.foster@mcgill.ca from the plan [9]. We will focus primarily on medication adherence, but recognize that poor adherence to other aspects of treatment, including attendance at clinic visits and blood testing are also very common [10] and impact outcomes.

In this paper, we will review the different forms of poor adherence, show that young people constitute a particularly high risk group, and consider the consequences and impact of poor adherence. We will also review factors that influence adherence, highlighting the most common barriers to adherence identified by young people, and consider different methods of measuring adherence. Finally, we will consider possible intervention strategies to improve adherence in young people.

\section{COMPONENTS OF ADHERENCE}

Excellent medication adherence requires taking the correct dose of medication, at the correct time of day, every day, for as long as the condition is being treated. There may be problems with any of these components of adherence (Table 1).

Dosing Adherence. Failure to take the correct dose of medication may occur for two possible reasons: dosing errors or deliberate modification of the dose by the patient. Dosing errors occur for a variety of reasons, including physician prescribing error, pharmacist dispensing error, poor communication between the patient and the physician or pharmacist, or misunderstanding on the part of the patient. Once identified, dosing errors are usually easily corrected. Deliberate dose modification by the patient is a more difficult problem to correct. Patients may reduce the dose of medication because they believe that the medication is too strong, because of unpleasant side effects, or because of financial constraints [11-13]. Patients who cannot afford a medication may take a lower dose of medication in an effort to make the supply last longer [14]. Dose reductions of more 
than $50 \%$ were associated with a $70 \%$ higher risk of graft failure compared with no reduction [15]. A dose higher than prescribed may also be consumed in an effort to make up for prior missed doses. Determining appropriateness of dosing requires open communication between the treating team and the patient, with the treating team directly acknowledging with the patient that patients may sometimes modify their doses, and that they want to understand when and why the patient may have done this.

Timing Adherence. Taking medications on schedule is referred to as 'timing adherence'. Incorrect timing of medication doses is the most common form of poor adherence; $76 \%$ of kidney transplant recipients between 11 and 20 years old indicated they had taken medications at least 1 hour late at least once in the prior week [16]. Some $46 \%$ of 250 adult kidney recipients had adherence issues, with $28 \%$ of those who had taken medications more than 2 hours late reporting that this happened more than once per week [17]. The consequences of off-schedule dosing, in terms of graft outcomes, are unknown. However, even minor deviations from the prescribed medication schedule were associated with significantly higher late acute rejection rates in heart [18] and kidney $[19,20]$ transplant recipients. It is unrealistic to expect that patients will always take their medications at exactly the same times every day. Most healthcare professionals are willing to accept a window of 12 hours around the expected dosing time [21, 22]. It is important, however, that physicians, nurses and pharmacists discuss the intended dosing schedule with transplant recipients and agree upon set dosing times. This approach will emphasize the importance of correct dose timing.

Taking Adherence. The proportion of prescribed doses of medication that are taken each day is referred to as 'taking adherence' [23]. In a study of 33 kidney transplant recipients 11-20 years old, $27 \%$ reported missing at least one dose of medication within the prior week [16]. Poor taking adherence has been linked to higher acute rejection and graft failure rates $[20,24]$. Like with timing adherence, there is no known safe level of taking adherence. More risky still than occasional missed doses are 'drug holidays', defined as missing $\geq 2$ consecutive doses [20,23]. Again, it is important that patients be made to feel comfortable disclosing missed doses of medications to the treating team to accurately inform clinical decision-making.

Persistence. Appropriate dosing, timing, and taking adherence are all examples of implementation or execution of the recommended treatment regimen [21, 22]. Continuing the medication regimen for as long as the condition being treated is present constitutes persistence [25]. Perhaps not surprisingly, complete discontinuation of immunosuppressive medications by transplant patients is not very common. Most studies showed persistence with immunosuppressive medications over the period of observation. However, discontinuation of study drug at 6 months after randomization was reported in $18.5 \%$ of patients taking once daily tacrolimus and $28 \%$ of patients taking twice daily tacrolimus in a trial of once daily versus twice daily tacrolimus dosing [21]. Discontinuation of medication was associated with an 8.3 times higher risk of graft failure among patients recorded in the USRDS covered under Medicare [15].
Multiple methods of assessing adherence should be used whenever feasible for optimal assessment of adherence in clinical and research settings as well as the advancement of scientific understanding of adherence.

\section{ADHERENCE AMONG YOUNG TRANSPLANT RECIPIENTS}

Adolescents and young adults with a broad range of chronic conditions, including diabetes, rheumatologic disorders, and organ transplantation have been shown to have poorer adherence to both medication and to general care (e.g., clinic appointments, routine blood monitoring) compared with other age groups [26-30]. This deterioration in adherence is likely related to the increasing independence afforded young people as they mature, with less time spent under direct adult supervision, and increasing responsibility placed on the young person for tasks related to medication taking and overall management of their condition [31-34].

Many prior studies of medication adherence in pediatric transplant recipients classified patients as either adherent or non-adherent. Different studies used different methods of measuring adherence, and slightly different definitions of non-adherence. In a systematic review of studies of immunosuppressive medication adherence, Dobbels estimated the prevalence of non-adherence at $30.7 \%$ overall [5]. When patient age was taken into account, the prevalence of non-adherence was estimated at $43.2 \%$ among adolescents ( $\geq 10$ years old), compared with $22.4 \%$ among younger children or a mixed younger and adolescent population. Dew and colleagues also found older age to be associated with poorer adherence in a meta-analysis of studies examining medical regimen adherence among pediatric solid organ transplant recipients, with non-adherence rates three times higher in adolescents than in younger children [4].

Although adolescents have shown a higher risk of poor adherence than younger children in the majority of studies, this finding is not universal. An American study of pediatric kidney transplant recipients found greater adherence, as measured using Medicare prescription claims data, among older patients than among younger ones [24]. This finding may have been influenced by the very select sample of eligible patients (only $22 \%$ of pediatric kidney transplant recipients are insured by Medicare) and/or by the method used to assess adherence. Compared with older kidney transplant recipients, those under 24 years old had a higher prevalence of poor adherence and a lower prevalence of high adherence in one U.S. study [6]; in another study the risk of poor adherence 12 months post-transplant was 1.7 times higher among those $0-18$ years and 1.6 times higher in those 19-24 years than among those 25-44 years old [15]. The evidence overall suggests that adolescents and young adults have the highest risk of poor adherence of any age group. This is also corroborated by clinical experience, and by the strong association between adolescent age and poorer graft outcomes [7,35]. Graft failure rates begin to rise at about 11 years of age, peak in the interval between 17 and 24 years, and decrease thereafter [7]. As illustrated in Fig. (1), the age at which graft failure rates are highest corresponds with the period during which adherence is likely poorest. 


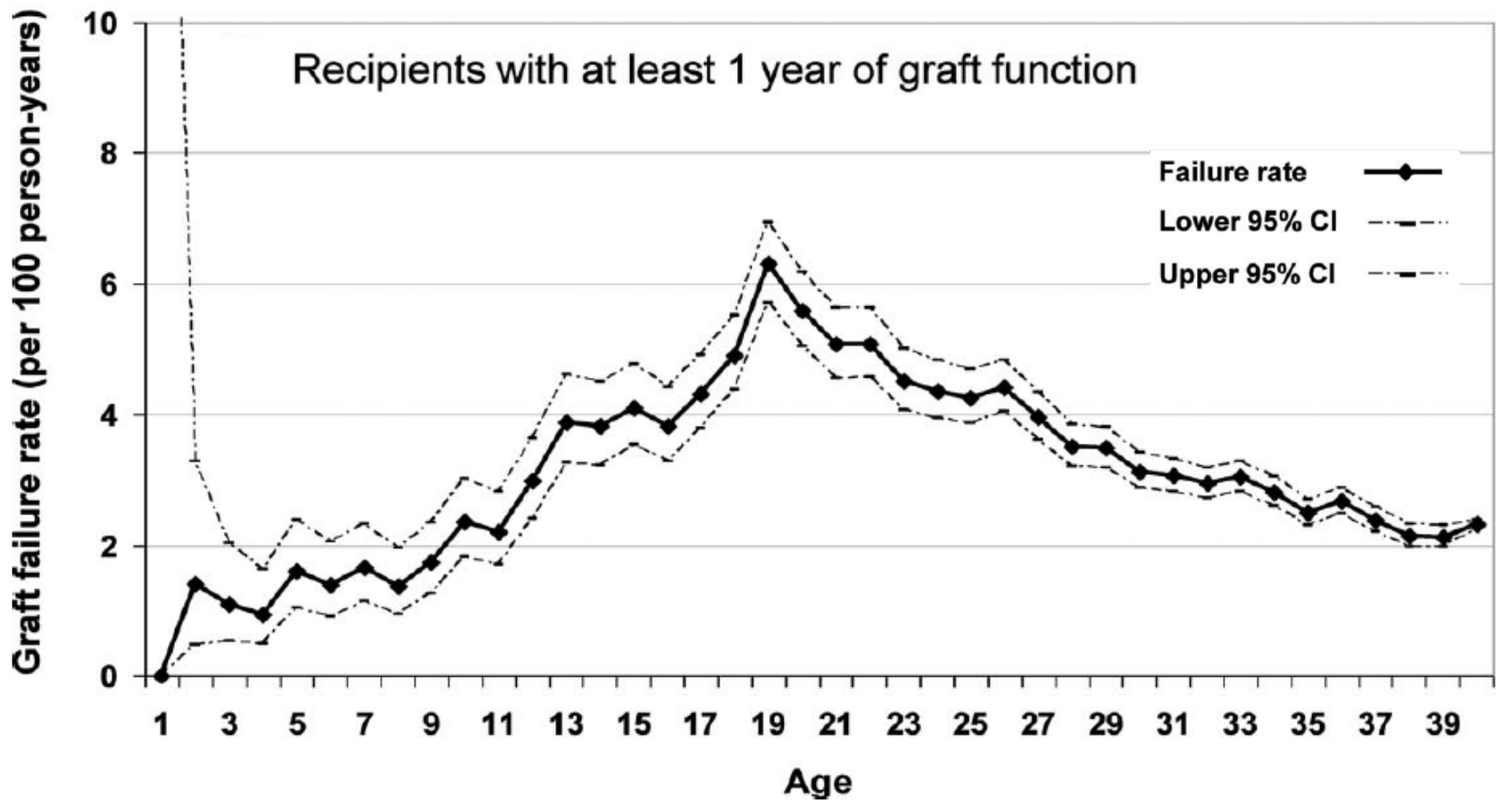

Fig. (1). Crude age-specific death-censored graft failure rates (failures per 100 person-years) in each 1-year age interval are shown with 95\% confidence intervals for kidney transplant recipients with at least 1 year of graft function (reproduced from [7]).

There is little information on how adherence behaviour prior to transplantation is used to assess transplant readiness or to identify patients at higher risk for poor adherence posttransplant. A recent survey of centers with membership in the United Network for Organ Sharing (response rate 44\%) found that only about half of respondents assessed adherence in any way before listing for transplant, and $94 \%$ used no formal questionnaire to assess medication adherence prior to listing [36]. The ability of medication adherence during dialysis to predict adherence behaviour post-transplant is also unknown. This may be particularly challenging in children and adolescents, whose behaviour is likely to change over time. More research is needed in this area.

\section{CONSEQUENCES AND IMPACT OF POOR ADHERENCE}

Numerous studies showed a strong association between poor adherence and adverse graft and patient outcomes. Among pediatric kidney transplant recipients for whom adherence was assessed using Medicare prescription claims, each $10 \%$ decrement in adherence was associated with an $8 \%$ higher hazard of graft failure [24]. Poor adherence was also associated with higher hospitalization and mortality rates in pediatric kidney transplant recipients [37]. There is growing evidence that poor medication adherence may be the most important mediator of late acute rejection and graft failure - especially among young people $[2,3,5,19,38,39]$. Antibody-mediated rejection is increasingly recognized as a major cause of graft failure among those whose grafts have survived for at least one year [3]. Sellarés and colleagues hypothesized that chronic poor adherence leads to donorspecific antibody formation and subsequent antibodymediated rejection - with a high risk of failure. In an observational study of 315 adult kidney transplant recipients, 60 experienced graft failure, of whom $47 \%$ had antibodymediated rejection on their last biopsy. The medical records contained concerns about poor adherence ten times more frequently among those whose grafts failed (32\%) than among those whose grafts did not fail (3\%) [3]. Another study of 628 adult kidney transplant recipients found that $48 \%$ of graft failures (death censored) that occurred $\geq 2$ years post-transplant could be attributed to poor adherence [2]. Of the 37 patients with evidence of poor adherence, $78 \%$ subsequently experienced graft failure, compared with only $7.8 \%$ of the 591 with good adherence. Younger age was significantly associated with a higher risk of graft failure due to poor adherence; among those $<50$ years old at transplant, $2 / 3$ of death censored graft failures were due to poor adherence [2].

Not all studies have shown a significant association between adherence and graft outcomes. A study of 243 adult kidney transplant recipients, in which adherence to a single immunosuppressive medication was monitored electronically during the first 6 months post-transplant, found no association between adherence and any of acute rejection, decline in glomerular filtration rate, or graft survival [40]. The authors hypothesized that sustained effects of immunosuppression induction agents may have protected patients from poor adherence during the study period. In addition, relatively good adherence in the period immediately following transplantation, large variability in causes of graft failure in this interval, and relatively low acute rejection rates may all have contributed to this finding. Another study of 121 prevalent adult kidney transplant recipients in whom adherence to a single immunosuppressive medication was monitored electronically for 12 months also found no association between adherence and any of acute rejection, decline 
in glomerular filtration rate, or graft loss [22]. However, poor outcomes were extremely uncommon in this cohort, suggesting that the study may have been underpowered to detect such associations.

Most agree that poor immunosuppression adherence is a major risk factor for graft failure. Graft failure has profound implications for both quality of life and patient longevity. Quality of life is significantly poorer for patients treated with dialysis than for those with a functioning transplant [41, 42]. Pediatric renal transplant recipients who returned to dialysis therapy following graft failure had a 4.4 times higher mortality rate than those who maintained graft function [43]. Individuals who lose a renal graft are not assured of receiving another; organ shortages and potential antibody sensitization related to the failed graft substantially limit opportunities for repeat transplantation.

In addition to the medical and quality of life costs associated with non-adherence, there are also substantial economic costs. The median annual cost per patient with graft function was estimated at $\$ 16844$, compared with $\$ 82$ 765 in the year of graft failure and $\$ 70581$ per year during dialysis therapy [44]. Another study estimated that persistent poor adherence is associated with a $\$ 12840$ increase in individual 3-year medical costs [6].

\section{DETERMINANTS OF ADHERENCE}

Fig. (2) illustrates the factors proposed by the World Health Organization (WHO) to determine treatment adherence [42]. These include patient-related factors (e.g. health beliefs, self-efficacy, knowledge, and perceived barriers to adherence), social and economic factors (e.g., family functioning, social supports, and medication costs), therapy-related factors (e.g., treatment side effects, duration of treatment, and regimen complexity), condition-related factors (e.g. symptoms, comorbidities, psychiatric conditions), and health care system-related and health care team-related factors [45]. Table 2 summarizes how the factors identified by the WHO fit into the framework proposed by Berben et al. to conceptualize the determinants of adherence at different "levels" [46]. Berben's framework emphasizes the fact that an individual patient's adherence is influenced not only by factors unique to that particular patient, but by the patient's interactions with those around him or her, and by the environment in which he or she is living. This framework includes patient-level (WHO patient, condition-, and therapy-related factors), "micro"-level (social factors and interactions with the care team), "meso"level (organization and expertise of the healthcare team and care processes), and "macro"-level (high-level healthcare systems factors, including care and medication cost coverage, and overall care environment) factors. When considering ways of improving adherence, clinicians often focus on what the patient must do; this framework helps highlight the things clinicians, and the healthcare system at large, may do to help support better adherence.

Table 1. Components of adherence.

\begin{tabular}{|c|c|c|}
\hline \multicolumn{2}{|c|}{ Component } & Potential Problems \\
\hline \multicolumn{2}{|l|}{ Persistence } & - Discontinuing medication \\
\hline \multirow{3}{*}{$\begin{array}{l}\text { Implementation/ } \\
\text { Execution }\end{array}$} & Taking & $\begin{array}{l}\text { - Missing doses intermittently or } \\
\text { consistently } \\
\text { - Drug holidays }\end{array}$ \\
\hline & Timing & $\begin{array}{l}\text { - Off-schedule dosing intermittently or } \\
\text { consistently }\end{array}$ \\
\hline & Dosing & $\begin{array}{l}\text { - Dosing errors } \\
\text { - Deliberate under- or over-dosing }\end{array}$ \\
\hline
\end{tabular}

Macro-level/Financial factors: International comparisons of adherence and graft outcomes may shed some light on the impact of heath care systems factors on adherence. A metaanalysis comparing European with American kidney transplant recipients found significantly poorer adherence among American patients [47]. The inability of some patients to pay for medications may have an important effect on adherence. In a survey of 254 American kidney transplant programs, $87 \%$ of adult programs and $67 \%$ of pediatric programs indicated that patients frequently contacted them with concerns about the high cost of immunosuppressive medications, and $75 \%$ of adult and $56 \%$ of pediatric

Table 2. Determinants of adherence.

\begin{tabular}{|l|l|l|}
\hline \multirow{2}{*}{ Level } & \multicolumn{1}{|c|}{ Factor } & \\
\hline \multirow{4}{*}{ Patient } & Sociodemographic & Sex, age, race, socioeconomic status \\
\cline { 2 - 4 } & Individual & Health beliefs, self-efficacy, condition and treatment knowledge, perceived barriers to adherence \\
\cline { 2 - 4 } & Condition-related & Nature of condition being treated, symptoms, comorbidities, psychiatric conditions \\
\cline { 2 - 4 } & Treatment related & Taste of medication, treatment side effects, duration of treatment, regimen complexity, number of doses per day \\
\hline \multirow{3}{*}{ Micro } & Social & Family structure, family functioning, social supports, economic factors \\
\cline { 2 - 4 } & Care team & Quality of interactions with healthcare team, trust in care providers \\
\hline \multirow{3}{*}{ Meso } & Care organization & Accessibility to care and to care providers \\
\cline { 2 - 4 } & Care team composition & Expertise of individuals on care team; inclusion of pharmacists, psychologists, nurses, physicians, surgeons \\
\cline { 2 - 4 } & Care processes & Methods of communication with care team; language barriers; clinic structure; frequency of visits; frequency of monitoring \\
\hline \multirow{2}{*}{ Macro } & Insurance & Medication and care coverage \\
\cline { 2 - 3 } & Healthcare system & General 'culture' of care; organization of healthcare \\
\hline
\end{tabular}




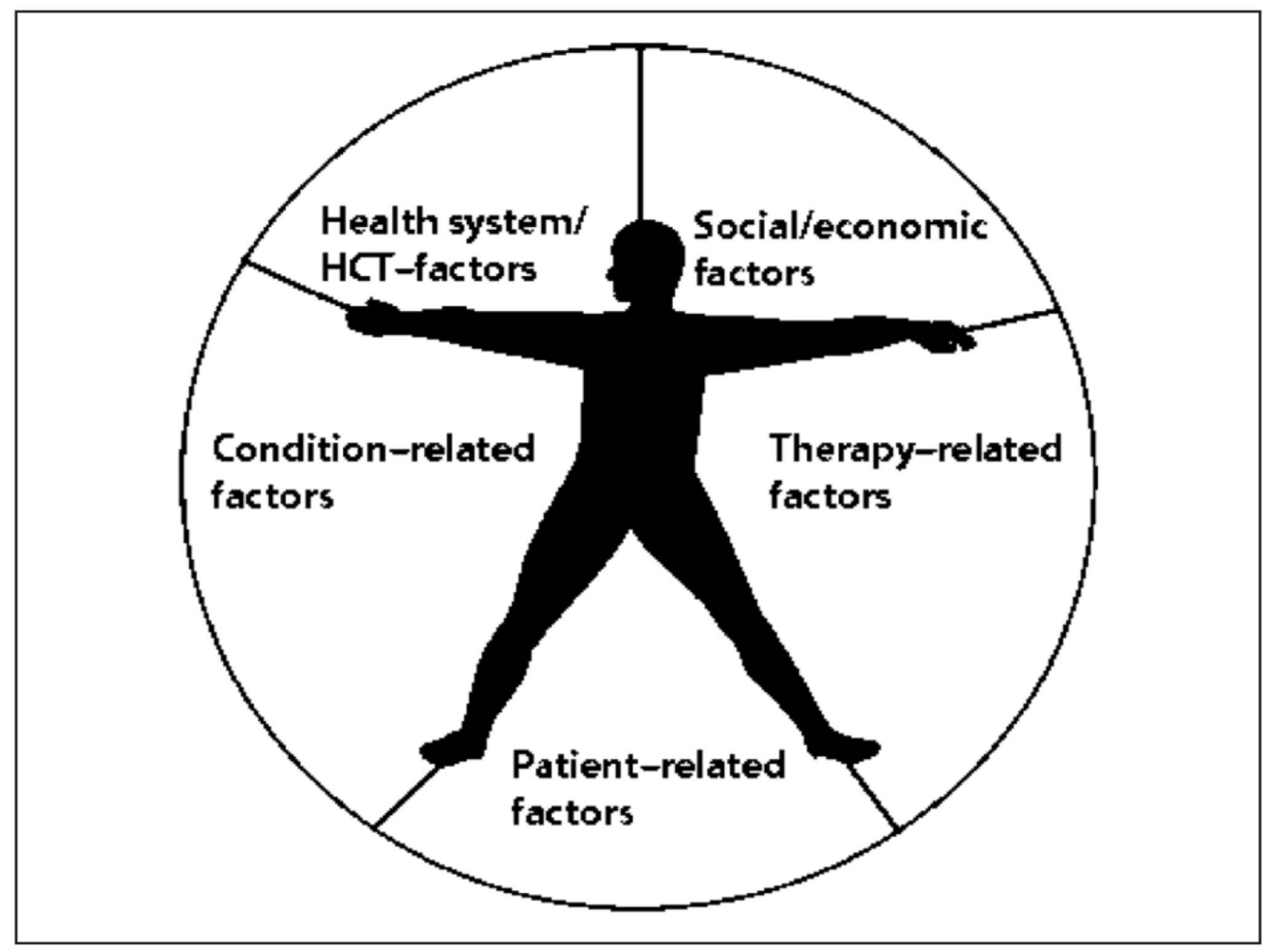

Fig. (2). Interacting factors influencing treatment adherence as identified by the World Health Organization (reproduced from [45]).

programs indicated that $>20 \%$ of their patients had trouble paying for their medications [13]. Furthermore, $43 \%$ of programs reported that $>10 \%$ patients do not take their medications as prescribed due to inability to pay for them. Some patients admit to reducing their doses to allow a prescription to last longer [14]. Inability to pay for medications appears to be more common among adults than children, likely due to additional options for medication cost coverage available to children. Loss of insurance coverage may be a contributing factor to the lower levels of adherence seen among young adults [48].

Meso-level factors: The role of healthcare processes and structures in adherence is just beginning to be explored. There is some evidence that adult-oriented healthcare processes may be poorly matched to the developmental needs of adolescents and young adults, contributing to poorer adherence in this group. There was a 2 -fold increased risk of graft failure during the period following transfer of Canadian pediatric kidney transplant recipients to adult care [49]. In a study of American pediatric kidney transplant recipients, the negative effect of transfer depended on age at transfer: individuals transferred at $<21$ years old had a $57 \%$ higher risk of graft failure than individuals of the same age who had been transferred at $\geq 21$ years old [50]. Transfer results not only in a change in care provider, but usually involves a major change in the care philosophy, practices, and resource availability. There are several important differences between the pediatric and adult care environments. Autonomy is expected in the adult care context, with emphasis being placed on the responsibility of the patient for their own health. In contrast, a more familycentered and paternalistic approach is common in the pediatric setting. In addition, the volume of patients per care provider is typically substantially higher in the adult versus pediatric care setting, resulting in less availability of adult care providers $[51,52]$.

Micro-level factors: Whereas social support has not been consistently identified as a significant determinant of adherence among adults $[11,22]$, lack of parental supervision and support, poor parent-patient communication, and poor family functioning have been identified as barriers to adherence among children and adolescents [4, 5, 51]. Family efficacy, defined as the family's ability to accomplish tasks needed to function, was associated with fewer perceived barriers to adherence [53]. Poor communication between the patient and the physician has also been shown to have a negative impact on adherence [54].

Patient-level factors: When considering patient-level determinants of adherence, it should be recognized that poorly adherent patients will each have a different combination of reasons for their poor adherence. Most poor adherence is 'unintentional' [14], and believed to be related to inadequate organizational skills and/or problem-solving abilities, or to complexity of the medical regimen. Forgetting was the most commonly stated reason for missing medications $(56 \%)$ in one study of adolescent renal transplant recipients [30], and the second most common $(29 \%)$, after organizational problems $(58 \%)$ in another study [55]. Breaches in adherence were most common when people were outside their normal routines [12, 14]. Evening doses were missed or late more frequently than morning doses [21, 22]. Other modifiable risk factors for poor adherence in adolescents with transplants include poor 
medication and disease knowledge and lack of a pillbox [4, $5,9,30,51,56]$. Depression has not been consistently identified as a significant determinant of adherence [11,57]. Interestingly, higher levels of anxiety were associated with better adherence among pediatric kidney and liver transplant recipients [57]. Self-efficacy, defined as a sense of control over one's environment and behaviour, has a positive influence on adherence, and was estimated to explain $9 \%$ of the variability in adherence in a study of 121 adult kidney transplant recipients [22].

Intentional poor adherence frequently relates to health beliefs, and may be more difficult to change. The Health Belief model suggests that people adhere to a treatment if they (i) perceive that they are susceptible to disease, (ii) believe that the disease has serious consequences, and (iii) believe that the benefits of taking action outweigh the costs associated with action and the barriers to taking action [11]. A survey of 558 adult kidney transplant recipients found that patients with good adherence had a stronger belief in the necessity of immunosuppressive medications than those with poorer adherence [11]. A study of kidney transplant recipients 11-20 years of age examined patients' perceptions of adversity associated with transplantation, hypothesizing that greater perceived adversity may tip the cost-benefit equation, favouring poor adherence. They found that those who received a transplant at $\geq 16$ years of age and those who had not experienced an interval of dialysis before transplantation perceived greater adversity with transplantation; however, there was no significant association between perceived adversity and adherence [16]. Among adolescents, poor adherence may reflect attempts to 'be normal' - though this represents less than $5 \%$ of reasons given for poor adherence [55].

Factors related to treatment may also influence adherence. Complexity of the medication regimen, multiple doses per day, and multiple pills per dose have all been identified as barriers to good adherence [5, 30, 58]. Some comparisons of the once-daily with the twice daily formulation of tacrolimus, including in a randomized trial [21], found greater satisfaction [59] and superior adherence with the once-daily formulation $[21,60,61]$. However, the proportion of patients who missed medication for at least one full day (i.e. a single dose for those on once-daily dosing or 2 consecutive doses for those on twice-daily dosing) was higher $(62 \%)$ among those on once-daily dosing than for those on twice-daily dosing (40\%). The consequences of missing a dose may be more serious with single daily dosing in terms of total drug exposure [62]. Furthermore, other studies found no difference in adherence between those taking once daily versus twice daily tacrolimus $[62,63]$.

Levels of adherence are also dynamic over time; it is common for adherence to decrease as time since transplant increases. Two studies of adult kidney transplant recipients found significant declines in adherence over time [20,64]. However, the independent effect of time since transplant is more difficult to assess in pediatric recipients, who are also moving into adolescence and young adulthood concurrently with increasing time since transplant.

\section{METHODS OF MEASURING ADHERENCE}

There is no perfect method of measuring medication adherence. Each method has advantages and disadvantage. Methods of measuring adherence, summarized in Table 3, can be divided into 'direct' and 'indirect' methods.

Direct Methods: Direct methods of measuring adherence include direct observation of medication taking - which is clearly impractical outside a hospital setting - and blood drug levels. Single trough levels of the immunosuppressive medications tacrolimus, cyclosporine, mycophenolate, and sirolimus that are low or undetectable may provide evidence of very recent poor adherence, but provide no information about adherence patterns over longer periods. Variability in trough levels of tacrolimus, as quantified by the standard deviation, is gaining popularity as a direct method of quantifying adherence among transplant recipients. High tacrolimus level standard deviation, which reflects erratic dosing, was strongly associated with number of acute rejections in adolescent liver transplant recipients [65], and decreased following an adherence-promoting intervention [66, 67]. A study of 144 heart, liver, kidney, and lung transplant recipients 8 to 18 years old found higher tacrolimus level standard deviations to be associated with a significantly higher risk of late acute rejection, and each 1 unit higher standard deviation of tacrolimus levels to be associated with a 1.58 times higher risk for graft loss [68]. Graft survival was better when the standard deviation was $<2.0$.

It has been suggested that the coefficient of variation may provide a better measure of variability in tacrolimus levels than standard deviation since higher standard deviation may reflect higher absolute levels of tacrolimus [69]. A study of 46 kidney transplant recipients $<22$ years old found that a coefficient of variation of tacrolimus levels greater than $41 \%$ was associated with a higher risk of rejection (odds ratio 9.7, $\mathrm{p}=0.005$ ). Some have suggested that it may be more appropriate to consider the proportion of tacrolimus trough levels outside the target range, or the mean deviation from target [70] rather than the standard deviation. This suggestion stems from the fact that target tacrolimus trough levels may change over time; if the dose is modified to reach a new target, and the level changes accordingly, the standard deviation will increase even in the face of excellent adherence.

There is some early evidence that standard deviation of sirolimus trough levels is also a reasonably good marker of adherence [71]. A composite of variability in tacrolimus or sirolimus trough levels and self-report, such as the 'system for integrated adherence monitoring' may also provide adherence information [71]. Unfortunately, the variability in the levels of other immunosuppressive medications are less useful. Variability in cyclosporin levels reflects adherence poorly [72]. Mycophenolic acid levels and azathioprine metabolites are not routinely monitored, and have not been studied. Although tracers may be added to medications in the research setting to provide a means of monitoring adherence directly, this is expensive and impractical for clinical purposes [73]. 
Table 3. Methods of measuring adherence.

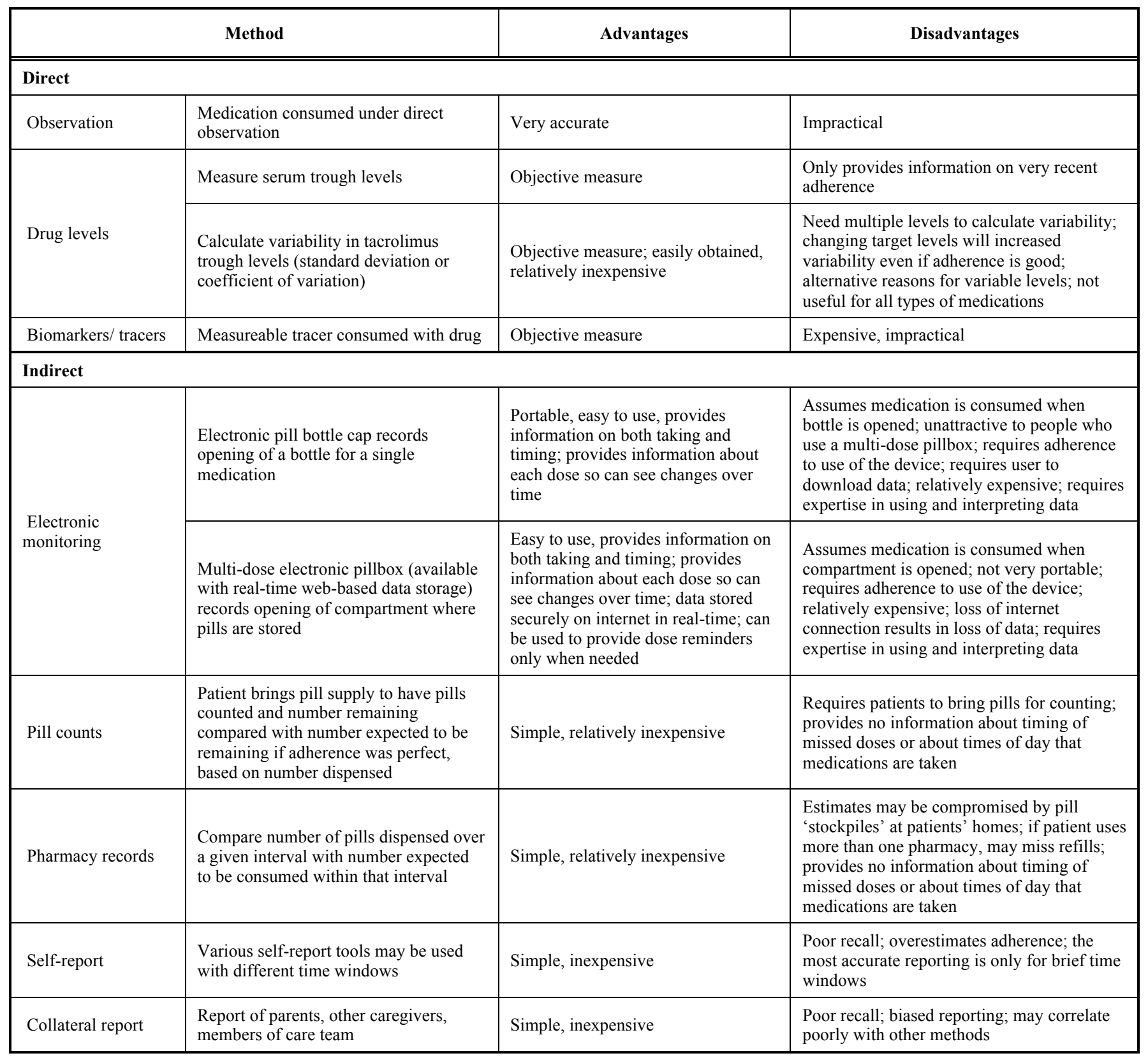

Indirect methods: Electronic monitoring is considered an indirect method, and makes the assumption that the medication is consumed every time the electronic device is opened. Medications may be monitored using a bottle with an electronic cap that records a date and time 'stamp' each time the bottle is opened (such as the Medication Event Monitoring System, MEMS) or using a multidose electronic pillbox such as the Vaica Simplemed device. Bottle devices have the advantage of being portable, but may be unacceptable to people who use a multidose pillbox to organize their medications [73], and require the user to bring the cap to the center for reading, or at least place it on a reader at home. Multidose electronic pillboxes are not very portable, and may depend on a reliable internet connection. However, they have several advantages, including real time storage of adherence data on a web-based system requiring no action on the part of the user, providing an organizational system that may itself promote adherence, and the option of providing reminders to users only if the device is not opened on time. All electronic monitors provide rich adherence information, allowing tracking of patterns of missed and late doses, and changes in adherence over time.

Pill counts are fairly simple, but require patients to bring pills for counting, and provide no information about the timing of missed doses or about times of day that medications are taken. Counts also assume that missing pills have been consumed. Pharmacy records can be used to estimate the number of pills consumed, knowing timing of refills and amount of medication dispensed at each refill. A significant limitation of pharmacy refill records is that patients may have 'stockpiles' of medication at home, left over after dose changes, making it difficult to accurately determine the amount of medication consumed. 
A variety of tools exist to capture adherence by selfreport. In general self-report tends to overestimate adherence. However, self-report does have moderate correlation with other methods, such as electronic monitoring [70]. The accuracy of self-report can be improved by remaining neutral and non-judgmental when questioning, and by limiting recall of adherence to a relatively short time period. Collateral report from parents or from members of the healthcare team may also be used. However the accuracy of collateral reports in diagnosing poor adherence is very limited [70].

\section{POTENTIAL INTERVENTIONS TO IMPROVE ADHERENCE}

Interventions to improve adherence may be applied at a program level, to all patients in the program, or at an individual level, targeted to patients identified to be at high risk. Program level interventions recognize that poor adherence is often unrecognized, and may include components targeting meso-, micro-, and patient-level factors influencing adherence. The goal of program level interventions is to improve the adherence of all patients in the program. The most effective intervention strategies likely include both program-level and targeted interventions. Prior work suggests that interventions administered repeatedly at regular intervals offer better sustained treatment effects than interventions delivered in single session or concentrated formats [74-78]. Repeated intervention sessions allow the opportunity to provide anticipatory guidance, recognizing that adherence barriers may change over time (e.g. during coming holidays or following a major change of routine such as leaving home for college) $[23,73]$.

An effective program-level intervention must address the most common, and most powerful, determinants of poor adherence. Prior studies of adult transplant recipients [38] and children and adolescents with other chronic illnesses [79-84] indicate that effective interventions include education in conjunction with some combination of adherence monitoring, promotion of problem-solving [82, 85-87], goal-setting, development of routines, and/or adherence support. There is some evidence suggesting that motivational interviewing techniques may also improve adherence [88, 89]. Other potential interventions at a program level include blood drug level and graft function monitoring at a higher frequency, and inclusion of a clinical pharmacist in the care team. Even something as simple as consistently asking about medication adherence at every clinic visit may have a positive effect on adherence. Given that organizational problems and 'forgetting' are two of the most commonly identified barriers to adherence, recommending and teaching use of a multidose pillbox may also help $[30,55]$. Routinely recommending a system of dose reminders, such as a watch or cellphone alarm, or an adherence system that provides phone or text message reminders, is another potentially useful strategy. A variety of smartphone applications and web-based tools to promote better self-care and medication adherence have become available in recent years. These include applications such as MyMedSchedule and the companion website MedAction
Plan (https://secure.medactionplan.com/ped/), and MyMeds (http://about.my-meds.com/), among others. A recent review of smartphone applications compares the features of many available products [90]. These applications provide features such as medication lists, adherence self-tracking, and text message dose reminders.

For adolescents and young adults, who are beginning to take over responsibility for their own care, it may also be useful to clearly identify who is responsible for each task related to medication taking, using a tool such as the Allocation of Responsibility for treatment regimen tasks questionnaire [34]. Tools designed to identify personal barriers to adherence, such as the Adolescent Medication Barriers Scale, may be used to tailor interventions to address the most relevant barriers [56]. Adherence support from a key person from outside the healthcare team - called a "personal trainer" [83, 84] has also shown promise in improving adherence as did a 'continuous self-improvement' intervention approach with adult kidney transplant recipients [91].

In order to effectively apply targeted interventions, high risk patients must first be identified. This may be done using adherence monitoring systems (such as electronic monitoring), following drug levels, using self-report tools, or based on prior behaviour (known poor adherence, rejection episodes). More frequent visits and bloodwork for patients at high risk may improve adherence [67]. Adherence 'contracts' have also demonstrated some efficacy in adult kidney transplant recipients [92].

\section{SUMMARY}

Adherence to immunosuppressive medications is essential to long term graft survival. However, long-term medication self-management and maximizing adherence is complex and difficult due to multiple and often interrelated factors. Categorizing these factors as patient (e.g., multiple medications, changes in routine), micro (e.g., poor family functioning), meso (e.g., changes in providers,) and macro (e.g., insurance coverage) factors provides a useful heuristic for guiding adherence assessment and intervention with patients. Optimizing graft survival and adherence in young people requires integrated and comprehensive care programs that include assessment, monitoring and intervention that directly considers factors on all of these levels (i.e., patient-, micro-, meso- and macro-). A number of empirically based intervention strategies focused on optimizing adherence are emerging in the literature. It will be essential that the dissemination of these tools occur as rapidly as possible to optimize the outcomes for young adults receiving transplants now and in the future.

\section{CONFLICT OF INTEREST}

The authors confirm that this article content has no conflict of interest.

\section{ACKNOWLEDGEMENTS}

Declared none. 


\section{REFERENCES}

[1] Bartosh SM, Ryckman FC, Shaddy R, Michaels MG, Platt JL, Sweet SC. A national conference to determine research priorities in pediatric solid organ transplantation. Pediatr Transplant 2008; 12(2): 153-66.

[2] Gaynor JJ, Ciancio G, Guerra G, et al. Graft failure due to noncompliance among 628 kidney transplant recipients with longterm follow-up: a single-center observational study. Transplantation 2014; 97(9): 925-33.

[3] Sellares J, de Freitas DG, Mengel M, et al. Understanding the causes of kidney transplant failure: the dominant role of antibodymediated rejection and nonadherence. Am J Transplant 2011; 12(2): 388-99.

[4] Dew MA, Dabbs AD, Myaskovsky L, et al. Meta-analysis of medical regimen adherence outcomes in pediatric solid organ transplantation. Transplantation 2009; 88(5): 736-46.

[5] Dobbels F, Ruppar T, De Geest S, Decorte A, Van DammeLombaerts R, Fine RN. Adherence to the immunosuppressive regimen in pediatric kidney transplant recipients: a systematic review. Pediatr Transplant 2010; 14(5): 603-13.

[6] Pinsky BW, Takemoto SK, Lentine KL, Burroughs TE, Schnitzler MA, Salvalaggio PR. Transplant outcomes and economic costs associated with patient noncompliance to immunosuppression. Am J Transplant 2009; 9(11): 2597-606.

[7] Foster BJ, Dahhou M, Zhang X, Platt RW, Samuel SM, Hanley JA. Association between age and graft failure rates in young kidney transplant recipients. Transplantation 2011; 92(11): 1237-43.

[8] Modi AC, Pai AL, Hommel KA, et al. Pediatric self-management: a framework for research, practice, and policy. Pediatrics 2012; 129(2): e473-85.

[9] Fine RN, Becker Y, De Geest S, et al. Nonadherence consensus conference summary report. Am J Transplant 2009; 9(1): 35-41.

[10] Shellmer DA, Dabbs AD, Dew MA. Medical adherence in pediatric organ transplantation: what are the next steps? Curr Opin Organ Transplant 2011; 16(5): 509-14.

[11] Chisholm-Burns M, Pinsky B, Parker G, et al. Factors related to immunosuppressant medication adherence in renal transplant recipients. Clin Transplant 2012; 26(5): 706-13.

[12] Tong A, Howell M, Wong G, Webster AC, Howard K, Craig JC. The perspectives of kidney transplant recipients on medicine taking: a systematic review of qualitative studies. Nephrol Dial Transplant 2011; 26(1): 344-54.

[13] Evans RW, Applegate WH, Briscoe DM, et al. Cost-related immunosuppressive medication nonadherence among kidney transplant recipients. Clin J Am Soc Nephrol 2010; 5(12): 2323-8.

[14] Griva K, Davenport A, Harrison M, Newman SP. Non-adherence to immunosuppressive medications in kidney transplantation: intent $v s$ forgetfulness and clinical markers of medication intake. Ann Behav Med 2012; 44(1): 85-93

[15] Takemoto SK, Pinsky BW, Schnitzler MA, et al. A retrospective analysis of immunosuppression compliance, dose reduction and discontinuation in kidney transplant recipients. Am J Transplant 2007; 7(12): 2704-11.

[16] Ratcliff MB, Blount RL, Mee LL. The relationship between adolescent renal transplant recipients' perceived adversity, coping, and medical adherence. J Clin Psychol Med Settings 2010; 17(2): 116-24.

[17] Lennerling A, Forsberg A. Self-reported non-adherence and beliefs about medication in a Swedish kidney transplant population. Open Nurs J 2012; 6: 41-6.

[18] De Geest S, Abraham I, Moons P, et al. Late acute rejection and subclinical noncompliance with cyclosporine therapy in heart transplant recipients. J Heart Lung Transplant 1998; 17(9): 854-63.

[19] Jarzembowski T, John E, Panaro F, et al. Impact of noncompliance on outcome after pediatric kidney transplantation: an analysis in racial subgroups. Pediatr Transplant 2004; 8(4): 367-71.

[20] Nevins TE, Thomas W. Quantitative patterns of azathioprine adherence after renal transplantation. Transplantation 2009; 87(5): 711-8.

[21] Kuypers DR, Peeters PC, Sennesael JJ, et al. Improved adherence to tacrolimus once-daily formulation in renal recipients: a randomized controlled trial using electronic monitoring. Transplantation 2013; 95(2): 333-40.

[22] Russell CL, Ashbaugh C, Peace L, et al. Time-in-a-bottle (TIAB): a longitudinal, correlational study of patterns, potential predictors, and outcomes of immunosuppressive medication adherence in adult kidney transplant recipients. Clin Transplant 2013; 27(5): E580-90.

[23] Denhaerynck K, Steiger J, Bock A, et al. Prevalence and risk factors of non-adherence with immunosuppressive medication in kidney transplant patients. Am J Transplant 2007; 7(1): 108-16.

[24] Chisholm-Burns MA, Spivey CA, Rehfeld R, Zawaideh M, Roe DJ, Gruessner R. Immunosuppressant therapy adherence and graft failure among pediatric renal transplant recipients. Am J Transplant 2009; 9(11): 2497-504

[25] Cramer JA, Roy A, Burrell A, et al. Medication compliance and persistence: Terminology and definitions. Value in Health 2008; 11(1): 44-47

[26] Annunziato RA, Emre S, Shneider B, Barton C, Dugan CA Shemesh E. Adherence and medical outcomes in pediatric liver transplant recipients who transition to adult services. Pediatr Transplant 2007; 11(6): 608-14.

[27] Crowley R, Wolfe I, Lock K, McKee M. Improving the transition between paediatric and adult healthcare: a systematic review. Arch Dis Child 2011; 96(6): 548-53.

[28] McDonagh JE. Transition of care from paediatric to adult rheumatology. Arch Dis Child 2007; 92(9): 802-7.

[29] Nakhla M, Daneman D, To T, Paradis G, Guttmann A. Transition to adult care for youths with diabetes mellitus: findings from a Universal Health Care System. Pediatrics 2009; 124(6): e1134-41.

[30] Zelikovsky N, Schast AP, Palmer J, Meyers KE. Perceived barriers to adherence among adolescent renal transplant candidates. Pediatr Transplant 2008; 12(3): 300-8.

[31] Bell L. Adolescents with renal disease in an adult world: meeting the challenge of transition of care. Nephrol Dial Transplant 2007; 22(4): 988-91.

[32] Bell LE, Bartosh SM, Davis CL, et al. Adolescent Transition to Adult Care in Solid Organ Transplantation: a consensus conference report. Am J Transplant 2008; 8(11): 2230-42.

[33] Pai AL, Ingerski LM, Perazzo L, Ramey C, Bonner M, Goebel J. Preparing for transition? The allocation of oral medication regimen tasks in adolescents with renal transplants. Pediatr Transplant 2010 ePub Sept. 30, 2010.

[34] Pai ALH, Gray E, Kurvival K, Ross J, Schoborg D, Goebel J. The Allocation of Treatment Responsibility Scale: A Novel Tool for Assessing Patient and Caregiver Management of Pediatric Medical Treatment Regimens. Pediatric Transplantation 2010; in press.

[35] Smith JM, Ho PL, McDonald RA. Renal transplant outcomes in adolescents: a report of the North American Pediatric Renal Transplant Cooperative Study. Pediatr Transplant 2002; 6(6): $493-$ 9.

[36] Brar A, Babakhani A, Salifu MO, Jindal RM. Evaluation of nonadherence in patients undergoing dialysis and kidney transplantation: United States transplantation practice patterns survey. Transplant Proc 2014; 46(5): 1340-6.

[37] Simons LE, Gilleland J, Blount RL, Amaral S, Berg A, Mee LL. Multidimensional Adherence Classification System: initial development with adolescent transplant recipients. Pediatr Transplant 2009; 13(5): 590-8.

[38] De Bleser L, Matteson M, Dobbels F, Russell C, De Geest S Interventions to improve medication-adherence after transplantation: a systematic review. Transpl Int 2009; 22(8): 78097.

[39] Prendergast MB, Gaston RS. Optimizing medication adherence: an ongoing opportunity to improve outcomes after kidney transplantation. Clin J Am Soc Nephrol 2010; 5(7): 1305-11.

[40] Israni AK, Weng FL, Cen YY, Joffe M, Kamoun M, Feldman HI. Electronically measured adherence to immunosuppressive medications and kidney function after deceased donor kidney transplantation. Clin Transplant 2011; 25(2): E124-31.

[41] Gerson AC, Furth SL, Neu AM, Fivush BA. Assessing associations between medication adherence and potentially modifiable psychosocial variables in pediatric kidney transplant recipients and their families. Pediatr Transplant 2004; 8(6): 543-50.

[42] Goldstein SL, Rosburg NM, Warady BA, et al. Pediatric end stage renal disease health-related quality of life differs by modality: a PedsQL ESRD analysis. Pediatr Nephrol 2009; 24(8): 1553-60.

[43] Foster BJ, Dahhou M, Zhang X, Platt RW, Hanley JA. Change in mortality risk over time in young kidney transplant recipients. Am J Transplant 2011; 11(11): 2432-42.

[44] System USRD. USRDS 2009 Annual Data Report: Atlas of Chronic Kidney Disease and End-Stage Renal Disease in the 
United States, Bethesda, MD: National Institutes of Health, National Institute of Diabetes and Digestive and Kidney Diseases; 2009.

[45] Sabate AL. Adherence to long-term therapies: Evidence for action. Geneva: World Health Organization; 2003.

[46] Berben L, Dobbels F, Engberg S, Hill MN, De Geest S. An ecological perspective on medication adherence. West J Nurs Res 2012; 34(5): 635-53.

[47] Denhaerynck K, Desmyttere A, Dobbels F, et al. Nonadherence with immunosuppressive drugs: U.S. compared with European kidney transplant recipients. Prog Transplant 2006; 16(3): 206-14.

[48] Callahan ST, Cooper WO. Continuity of health insurance coverage among young adults with disabilities. Pediatrics 2007; 119(6): $1175-80$

[49] Samuel SM, Nettel-Aguirre A, Hemmelgarn BR, et al. Graft Failure and Adaptation Period to Adult Healthcare Centers in Pediatric Renal Transplant Patients. Transplantation 2011; 91(12): $1380-5$.

[50] Foster BJ, Platt RW, Dahhou M, Zhang X, Bell LE, Hanley JA. The impact of age at transfer from pediatric to adult-oriented care on renal allograft survival. Pediatr Transplant 2011; 15(7): 750-9.

[51] Dobbels F, Van Damme-Lombaert R, Vanhaecke J, De Geest S. Growing pains: non-adherence with the immunosuppressive regimen in adolescent transplant recipients. Pediatr Transplant 2005; 9(3): 381-90.

[52] Wolff G, Strecker K, Vester U, Latta K, Ehrich JH. Noncompliance following renal transplantation in children and adolescents. Pediatr Nephrol 1998; 12(9): 703-8.

[53] Guilfoyle SM, Goebel JW, Pai AL. Efficacy and flexibility impact perceived adherence barriers in pediatric kidney posttransplantation. Fam Syst Health 2011; 29(1): 44-54.

[54] Delucchi A, Gutierrez H, Arrellano P, Slater C, Meneses M, Lopez I. Factors that influence nonadherence in immunosuppressant treatment in pediatric transplant recipients: a proposal for an educational strategy. Transplant Proc 2008; 40(9): 3241-3.

[55] Simons LE, McCormick ML, Mee LL, Blount RL. Parent and patient perspectives on barriers to medication adherence in adolescent transplant recipients. Pediatr Transplant 2009; 13(3): 338-47.

[56] Simons LE, McCormick ML, Devine K, Blount RL. Medication Barriers Predict Adolescent Transplant Recipients' Adherence and Clinical Outcomes at 18-Month Follow-up. J Pediatr Psychol 2010.

[57] Wu YP, Aylward BS, Steele RG. Associations between internalizing symptoms and trajectories of medication adherence among pediatric renal and liver transplant recipients. J Pediatr Psychol 2009; 35(9): 1016-27.

[58] Morales JM, Varo E, Lazaro P. Immunosuppressant treatment adherence, barriers to adherence and quality of life in renal and liver transplant recipients in Spain. Clin Transplant 2012; 26(2): $369-76$.

[59] Hatakeyama S, Fujita T, Yoneyama T, et al. A switch from conventional twice-daily tacrolimus to once-daily extended-release tacrolimus in stable kidney transplant recipients. Transplant Proc 2012; 44(1): 121-3.

[60] Obi Y, Ichimaru N, Kato T, et al. A single daily dose enhances the adherence to immunosuppressive treatment in kidney transplant recipients: a cross-sectional study. Clin Exp Nephrol 2012; 17(2): 310-5.

[61] Nakamura Y, Hama K, Katayama H, et al. Safety and efficacy of conversion from twice-daily tacrolimus (prograf) to once-daily prolonged-release tacrolimus (graceptor) in stable kidney transplant recipients. Transplant Proc 2012; 44(1): 124-7.

[62] Hougardy JM, de Jonge H, Kuypers D, Abramowicz D. The oncedaily formulation of tacrolimus: a step forward in kidney transplantation? Transplantation 2012; 93(3): 241-3.

[63] Pape L, Heidotting N, Ahlenstiel T. Once-daily tacrolimus extended-release formulation: 1 year after conversion in stable pediatric kidney transplant recipients. Int J Nephrol 2011; 2011: 126251.

[64] Couzi L, Moulin B, Morin MP, et al. Factors predictive of medication nonadherence after renal transplantation: a French observational study. Transplantation 2013; 95(2): 326-32.

[65] Shemesh E, Shneider BL, Savitzky JK, et al. Medication adherence in pediatric and adolescent liver transplant recipients. Pediatrics 2004; 113(4): 825-32.
[66] Miloh T, Annunziato R, Arnon R, et al. Improved adherence and outcomes for pediatric liver transplant recipients by using text messaging. Pediatrics 2009; 124(5): e844-50.

[67] Shemesh E, Annunziato RA, Shneider BL, et al. Improving adherence to medications in pediatric liver transplant recipients. Pediatr Transplant 2008; 12(3): 316-23.

[68] Pollock-Barziv SM, Finkelstein Y, Manlhiot C, et al. Variability in tacrolimus blood levels increases the risk of late rejection and graft loss after solid organ transplantation in older children. Pediatr Transplant 2010; 14(8): 968-75.

[69] Hsiau M, Fernandez HE, Gjertson D, Ettenger RB, Tsai EW. Monitoring nonadherence and acute rejection with variation in blood immunosuppressant levels in pediatric renal transplantation. Transplantation 2011; 92(8): 918-22.

[70] Schafer-Keller P, Steiger J, Bock A, Denhaerynck K, De Geest S. Diagnostic accuracy of measurement methods to assess nonadherence to immunosuppressive drugs in kidney transplant recipients. Am J Transplant 2008; 8(3): 616-26.

[71] Pai AL, Rausch J, Tackett A, Marsolo K, Drotar D, Goebel J. System for integrated adherence monitoring: real-time nonadherence risk assessment in pediatric kidney transplantation. Pediatr Transplant 2012; 16(4): 329-34.

[72] Cakaloglu Y, Tredger JM, Devlin J, Williams R. Importance of cytochrome P-450IIIA activity in determining dosage and blood levels of FK 506 and cyclosporine in liver transplant recipients. Hepatology 1994; 20(2): 309-16.

[73] Zelikovsky N, Schast AP. Eliciting accurate reports of adherence in a clinical interview: development of the Medical Adherence Measure. Pediatr Nurs 2008; 34(2): 141-6.

[74] Chisholm MA, Mulloy LL, Jagadeesan M, DiPiro JT. Impact of clinical pharmacy services on renal transplant patients' compliance with immunosuppressive medications. Clin Transplant 2001; 15(5): 330-6.

[75] Klein A, Otto G, Kramer I. Impact of a pharmaceutical care program on liver transplant patients' compliance with immunosuppressive medication: a prospective, randomized, controlled trial using electronic monitoring. Transplantation 2009; 87(6): 839-47.

[76] Dejean NB, Rontaing L, Lapeyre-Mestre M, Roge B, Durand D. Educational program to reduce noncompliance after renal transplantation. In: European Renal Association and European Dialysis and Transplantation Association; 2004; Lisbon, Portugal; 2004.

[77] Fennell RS, Foulkes LM, Boggs SR. Family-based program to promote medication compliance in renal transplant children. Transplant Proc 1994; 26(1): 102-3.

[78] De Geest S, Schafer-Keller P, Denhaerynck K, et al. Supporting medication adherence in renal transplantation (SMART): a pilot RCT to improve adherence to immunosuppressive regimens. Clin Transplant 2006; 20(3): 359-68.

[79] Bonner S, Zimmerman BJ, Evans D, Irigoyen M, Resnick D, Mellins RB. An individualized intervention to improve asthma management among urban Latino and African-American families. J Asthma 2002; 39(2): 167-79.

[80] Smith NA, Seale JP, Ley P, Shaw J, Bracs PU. Effects of intervention on medication compliance in children with asthma. Med J Aust 1986; 144(3): 119-22.

[81] Hovell MF, Sipan CL, Blumberg EJ, et al. Increasing Latino adolescents' adherence to treatment for latent tuberculosis infection: a controlled trial. Am J Public Health 2003; 93(11): 1871-7.

[82] Rapoff MA, Belmont J, Lindsley C, Olson N, Morris J, Padur J. Prevention of nonadherence to nonsteroidal anti-inflammatory medications for newly diagnosed patients with juvenile rheumatoid arthritis. Health Psychol 2002; 21(6): 620-3.

[83] Nansel TR, Iannotti RJ, Simons-Morton BG, Plotnick LP, Clark LM, Zeitzoff L. Long-term maintenance of treatment outcomes: diabetes personal trainer intervention for youth with type 1 diabetes. Diabetes Care 2009; 32(5): 807-9.

[84] Nansel TR, Iannotti RJ, Simons-Morton BG, et al. Diabetes personal trainer outcomes: short-term and 1-year outcomes of a diabetes personal trainer intervention among youth with type 1 diabetes. Diabetes Care 2007; 30(10): 2471-7.

[85] Laffel LM, Vangsness L, Connell A, Goebel-Fabbri A, Butler D, Anderson BJ. Impact of ambulatory, family-focused teamwork 
intervention on glycemic control in youth with type 1 diabetes. $\mathrm{J}$ Pediatr 2003; 142(4): 409-16.

[86] Quittner AL, Drotar D, Ievers-Landis C, Seidner D, Slocum N, Jacobson J. Adherence to medical treatments in adolescents with cystic fibrosis: The development and evalutation of family-based interventions. In: Drotar D, editor. Promoting adherence to medical treatment in childhood chronic illness: Concepts, Methods, and Interventions. Mahwah, N.J.: Lawrene Erlbaum Associates; 2000. p. 383-361.

[87] Quittner AL, Modi AC, Roux AL. Psychosocial challenges and clinical interventions for children with cystic fibrosis: A developmental approach. In: Brown R, editor. Handbook of Pediatric Psychology in School Settings. New Jersey: Lawrence Erlbaum Associates; 2003. p. 333-361.

[88] Hardeman W, Lamming L, Kellar I, et al. Implementation of a nurse-led behaviour change intervention to support medication taking in type 2 diabetes: beyond hypothesised active ingredients (SAMS Consultation Study). Implement Sci 2014; 9: 70.
[89] Milne S, Orbell S, Sheeran P. Combining motivational and volitional interventions to promote exercise participation: protection motivation theory and implementation intentions. $\mathrm{Br} \mathrm{J}$ Health Psychol 2002; 7(Pt 2): 163-84.

[90] Dayer L, Heldenbrand S, Anderson P, Gubbins PO, Martin BC. Smartphone medication adherence apps: potential benefits to patients and providers. J Am Pharm Assoc (2003) 2013; 53(2): 172-81.

[91] Russell C, Conn V, Ashbaugh C, Madsen R, Wakefield M, Webb A, et al. Taking immunosuppressive medications effectively (TIMELink): a pilot randomized controlled trial in adult kidney transplant recipients. Clin Transplant 2011; 25(6): 864-70.

[92] Chisholm-Burns MA, Spivey CA, Graff Zivin J, Lee JK, Sredzinski E, Tolley EA. Improving outcomes of renal transplant recipients with behavioral adherence contracts: a randomized controlled trial. Am J Transplant 2013; 13(9): 2364-73.

Received: September 25, 2014

Revised: October 1, 2014

Accepted: October 2, 2014

(C) Foster and Pai; Licensee Bentham Open.

This is an open access article licensed under the terms of the Creative Commons Attribution Non-Commercial License (http://creativecommons.org/licenses/by-nc/3.0/) which permits unrestricted, non-commercial use, distribution and reproduction in any medium, provided the work is properly cited. 